\title{
openheart Clinical use of 4D flow MRI for quantification of aortic regurgitation
}

\author{
Ana Alvarez (1) , ${ }^{1,2}$ Vicente Martinez, ${ }^{1}$ Gonzalo Pizarro, ${ }^{2,3}$ Manuel Recio, \\ Jose Ángel Cabrera ${ }^{4}$
}

To cite: Alvarez A, Martinez V, Pizarro G, et al. Clinical use of 4D flow MRI for quantification of aortic regurgitation. Open Heart 2020;7:e001158. doi:10.1136/ openhrt-2019-001158

Received 27 August 2019 Revised 27 December 2019 Accepted 7 January 2020

\section{Check for updates}

(C) Author(s) (or their employer(s)) 2020. Re-use permitted under CC BY-NC. No commercial re-use. See rights and permissions. Published by BMJ.

${ }^{1}$ Imaging Department, Hospital Universitario Quironsalud Madrid, Madrid, Spain

${ }^{2}$ Universidad Europea de Madrid, Madrid, Spain

${ }^{3}$ Department of Cardiology, Complejo Hospitalario Ruber Juan Bravo, Madrid, Spain ${ }^{4}$ Department of Cardiology, Hospital Universitario Quironsalud Madrid, Madrid, Madrid, Spain

Correspondence to Dr Ana Alvarez;

anaalvarezvazquez@gmail.com

\section{ABSTRACT}

Objective The main objective of the present study was to compare the use of four-dimensional (4D) flow MRI with the habitual sequence (two-dimensional phase-contrast (2DPC) MRI) for the assessment of aortic regurgitation (AR) in the clinical routine.

Methods This was a retrospective, observational cohort study of patients with varying grades of AR. For the purposes of the present study, we selected all the cases with a regurgitant fraction (RF) $>5 \%$ as determined by 2DPC MRI $(n=34)$. In all cases, both sequences (2DPC and $4 \mathrm{D}$ flow MRI) were acquired in a single session to ensure comparability. We compared the results of the two techniques by evaluating forward flow, regurgitant flow and regurgitation fraction. Then, the patients were divided into subgroups to determine if these factors had any influence on the measurements: aortic diameter ( $\leq$ vs $>38 \mathrm{~mm}$ ), valve anatomy (tricuspid vs bicuspid/ quadricuspid), stenosis (gradient $\geq 15 v s<15$ ) and region of interest location (aortic valve vs sinotubular junction). Results No statistically significant differences were observed between the two techniques with Pearson's correlation coefficients $(r)$ of forward flow $(r=0.826 / p$ value $<0001)$, regurgitant flow $(r=0.866 / p$ value $<0001)$ and $R F(r=0.761 / p$ value $<0001)$.

Conclusions The findings of this study confirm the value of $4 \mathrm{D}$ flow MRI for grading AR in clinical practice with an excellent correlation with the standard technique (2DPC MRI).

\section{INTRODUCTION}

Aortic regurgitation (AR) is the diastolic flow of blood from the aorta to the left ventricle (LV), which leads to volume and pressure overload on the LV. According to recent clinical guidelines, ${ }^{12}$ echocardiography is the imaging technique of choice for the diagnosis, quantification and follow-up of AR.

Echocardiography provides data on the most relevant prognostic parameters-LV dilatation and/or dysfunction-needed to monitor patients with AR. ${ }^{1-3}$

MRI is considered a complementary technique in patients with AR with inadequate echocardiographic image quality or discrepant results. MRI provides more precise estimates of LV volumes, function and

\section{Key messages}

What is already known about this subject?

- Four-dimensional (4D) flow MRI allows to measure non-laminar flow in any direction in space, with flow encoding in all three spatial directions along the cardiac cycle, overcoming one of the several limitations of two-dimensional, phase-contrast (2DPC) MRI. Several recent studies have verified the validity of 4D flow MRI compared with both echocardiography and 2DPC MRI. Some authors found that 4D flow MRI and echocardiography presented good interobserver correlation, with good agreement in assessing valvular regurgitation. Flow and velocity measurements obtained by 4D flow MRI in mediastinal vessels have been successfully compared in healthy patients.

What does this study add?

- To our knowledge, despite the prevalence of aortic regurgitation (AR), there is not a study comparing 4D flow MRI with 2DPC MRI for grading AR in routine clinical practice. We found that there is a good correlation between the two sequences to quantify aortic positive and negative flow and aortic regurgitant fraction.

How might this impact on clinical practice?

- From our point of view, when prospective studies also verified the prognostic information obtained from 4D flow MRI, this sequence will substitute 2DPC MRI in the clinical protocol of cardiac magnetic resonance (CMR). We have seen its good correlation with the habitual sequence for grading $A R$, one of the most common valvular pathologies studied with MRI. Also, with the numerous advantages of the sequence, the indications of MRI will increase in valvular pathologies.

mass, and aortic dilatation than echocardiography. ${ }^{145}$

Studies have shown that the regurgitant fraction (RF) can be directly measured using twodimensional, phase-contrast (2DPG MRI), whose diagnostic capacity has been known since $1960 .{ }^{6}$ 2DPC MRI can also measure flow, velocity and direction accurately and reproducibly, ${ }^{7}$ even considering the limitations ${ }^{8}$ of 2DPC MRI (eg, the assumption of laminar 
flow and a dependency on the flow intercept angle). In fact, some studies have shown that 2DPC MRI could be superior to transthoracic echography in quantifying AR for prognostic purposes. ${ }^{9-11}$

For this reason, 2DPC MRI is included in routine MRI protocols.

Three-dimensional (3D) flow MRI is a technique known around for 20 years but recent developments in image acquisition protocols, as well as dedicated postprocessing tools, have contributed to the recent implementation of this approach in clinical workflow.

3D flow MRI with these new developments, known nowadays as four-dimensional (4D) flow MRI (3D within the time of the cardiac cycle), has emerged as an alternative clinical approach to measuring blood flow. The clinical validity of $4 \mathrm{D}$ flow MRI to evaluate several relevant parameters has been demonstrated, although additional studies are needed to confirm its capacity to assess other parameters such as wall shear stress. ${ }^{12}$ This technique allows to measure non-laminar flow in any direction in space, with flow encoding in all three spatial directions along the cardiac cycle, overcoming one of the several limitations of 2DPC MRI (single velocity encoding direction perpendicular to 2D plane), thus offering new possibilities for MRI in the assessment of AR.

Several recent studies ${ }^{13-15}$ have verified the validity of 4D flow MRI compared with both echocardiography and 2DPC MRI. Hsiao et $a l^{16}$ found that 4D flow MRI and echocardiography presented good interobserver correlation, with good agreement in assessing valvular regurgitation. Flow and velocity measurements obtained by $4 \mathrm{D}$ flow MRI in mediastinal vessels have been successfully compared in healthy patients ${ }^{16}$ and in patients with congenital heart disease. ${ }^{17}$

To our knowledge, despite the prevalence of AR, there is not a study comparing 4D flow MRI with 2DPC MRI for grading AR in routine clinical practice.

In this context, the main objective of the present study was to compare the use of $4 \mathrm{D}$ flow MRI with the habitual sequence (2DPC MRI) (already validated compared with ultrasound) for the assessment of AR in the clinical routine. More specifically, our aim was to determine if these two imaging modalities are equivalent with regard to AR quantification.

We hypothesised that the regurgitant flow data obtained by 4D flow MRI would not differ significantly from that obtained by 2DPC MRI. Furthermore, we expected that 4D flow MRI would permit an accurate grading of AR and would replace 2DPC in the clinical cardiac MRI protocol.

Secondary objectives were to determine whether anatomical variations (aortic diameter, valve anatomy) or other variables (presence of stenosis, location of region of interest (ROI)) affect the comparability of the quantification of AR severity with the two sequences.

\section{MATERIAL AND METHODS}

Type of study

This was a retrospective, observational cohort study of patients with varying grades of AR. All participants were treated at the Quirónsalud Madrid University Hospital in Madrid, Spain.

This study received no funding.

\section{Study population}

We retrospectively analysed the medical records of 220 patients who underwent both 4D flow MRI and 2DPC MRI between May 2017 and March 2018 at our institution.

For the purposes of the present study, we selected all the cases with an RF $>5 \%$ as determined by 2DPC MRI $(\mathrm{n}=34)$.

Exclusion criteria were as follows: (1) aortic valve or root replacement and (2) poor quality imaging studies in which the 2DPC and/or 4D flow sequence was not evaluable, or in which the studies were incomplete (patient failure to complete the test or refusal to accept intravenous contrast).

All patients in the study met the relevant clinical criteria for MRI. Prior to study inclusion, all patients completed a questionnaire to provide data on medical and surgical history, medications, allergies and implants (eg, pacemaker or metal implants). The study procedure was explained in detail to the patients, including the risks and the test duration, prior to obtaining signed informed consent.

\section{MRI protocol}

The MRI studies were performed using the GE Signa 1.5T MRI (GE Medical Systems, Milwaukee, Wisconsin, USA) with a 32-channel surface coil. As part of standard procedures, most of the imaging studies included standard cine and delayed-enhancement MRI sequences to assess for the presence of myocardial fibrosis. ${ }^{18}$ In all cases, both sequences (2DPC and 4D flow MRI) were acquired in a single session to ensure comparability.

\section{DPC MRI}

Acquisition

For image acquisition of the 2DPC MRI sequence, we used a single double oblique planned acquisition plane orthogonal to the wall of the aorta. There is some disagreement with regard to the optimal imaging plane to assess AR. Some authors propose placing the plane at the sinotubular junction or the proximal ascending aorta, ${ }^{19}$ while other authors advocate placement at the middle ascending aorta ${ }^{15}$ or below the aortic valve. ${ }^{20}$ At our institution, the slices are placed at the valve or the sinotubular junction, depending on the anatomical characteristics of the patient.

A fast, two-dimensional sequence was used with the patient in end-expiratory breath-hold with retrospective cardiac gating (30 reconstructed phases per cardiac cycle) and $k$-space segmentation of four views/segment. 
Other parameters were as follows: matrix, 192×128; field of view, $420 \times 294 \mathrm{~mm}$ and slice thickness, $5 \mathrm{~mm}$ (without spacing). As we used a fixed four views/segment, the true temporal resolution in data acquisition is $45.6 \mathrm{~ms}$. Repeat and echo times were 5.7 and $3.5 \mathrm{~ms}$, respectively, with a $15^{\circ}$ flip angle. The acquisition time was approximately $14 \mathrm{~s}$. In all cases, velocity encoding (VENC) was initially set at $150-200 \mathrm{~cm} / \mathrm{s}$ and progressively increased by $50 \mathrm{~cm} / \mathrm{s}$ until the image contained no artefacts. The lowest VENC at which no velocity aliasing is observed was selected. ${ }^{8}$

\section{Processing}

The data were processed with the CardiacVX software for GE Advantage Workstation V.4.4 (GE Healthcare, USA), which offers a semiautomatic analysis in the plane acquired during the test. An experienced radiologist $(>11$ years of experience), working in collaboration with a more experienced radiologist ( $>35$ years of experience), place an ROI at the aorta in the acquired plane, verifying that the propagation adapts to the anatomical limits, to obtain a flow-time curve.

\section{D flow MRI}

\section{Acquisition}

Prior to sequencing, a peripheral intravenous line is started to administer the gadolinium-based (Gadobutrol) contrast agent (Gadovist $1 \mathrm{mmol} / \mathrm{mL}$; Bayer, Mijdrecht, The Netherlands). Image acquisition begins simultaneously with the injection of $0.15 \mathrm{mmol} / \mathrm{kg}$ of contrast agent followed immediately by saline flush. The dose of the contrast agent is based on current recommendations of the Society for Cardiovascular Magnetic Resonance. ${ }^{21}$

The use of contrast is not necessary but allows faster scanning. We use gadolinium-based agent in our clinical MRI protocol to obtain late gadolinium enhancement imaging. Acquiring the 4D flow CMR data after the study that requires contrast administration takes advantage of the enhanced signal-to-noise ratio and thus velocityto-noise ratio, as well as contrast between blood and surrounding tissue. ${ }^{12}$

A 3D volume is acquired from the cardiac apex to the aortic arch with the patient in free-breathing and retrospective ECG-cardiac gating. The sequence adapts the views/segment automatically based on patient heart rate (HR) (smaller with $\mathrm{HR}$ and vice versa) to ensure 16 cardiac phases are acquired per cardiac cycle. The reconstructed images are linearly interpolated into 30 cardiac phases for better visualisation of the temporal variation. The acquisition time ranges from 7 to $10 \mathrm{~min}$.

In all cases, VENC was the same as that used in the 2DPC MRI. The technical characteristics of this sequence are summarised in table 1 .

\section{Processing}

The raw data are sent directly to a cloud software application (Arterys, San Francisco, California, USA) for image reconstruction and data correction. This application also
Table 1 Acquisition parameters in 4D flow MRI

\begin{tabular}{ll}
\hline Acquired resolution $(\mathrm{mm})$ & $2.78 \times 2.78 \times 2.40(\mathrm{RL} \times \mathrm{AP} \times \mathrm{IS})$ \\
\hline Reconstructed resolution $(\mathrm{mm})$ & $1.95 \times 1.95 \times 1.20(\mathrm{ZIP} \times 2)$ \\
\hline Average scan time (min:s) & $7: 30$ \\
\hline Average VENC $(\mathrm{cm} / \mathrm{s})$ & 160 \\
Views per segment & Adaptative to patient HR \\
FA & $15^{\circ}$ \\
TR & 4.25 \\
TE & 2.25 \\
Phases to reconstruct & 30
\end{tabular}

FA, flip angle; HR, heart rate; TE, echo time; TR, repetition time; VENC, velocity encoding.

provides real-time, 3D postprocessing tools to quantify function and flow. This image analysis is anonymised, and both automatic and semiautomatic analyses are available. The flow is visualised with a superimposed colour code based on the velocity of the anatomical data. Tools are available to define flow direction and trajectory (streamlines and vectors).

For the quantitative analysis, the same two radiologistswithout access to the 2DPC MRI analysis or the patient's personal data-place the ROI (by consensus agreement) in a double plane orthogonal to the ascending aorta in the same plane used for 2DPC MRI acquisition. The ROI propagates throughout the cardiac cycle (automatic or manual corrections can be made in each frame if necessary), thus verifying that the ROI adapts to the vessel in all 30 phases of the cardiac cycle.

\section{Comparison: 2DPC MRI versus 4D flow MRI}

We compared the overall results of the two techniques by evaluating three variables: forward flow, regurgitant flow and RF. Also, we compare the grading (based on RF) between the two techniques.

Then, the patients were divided into subgroups to determine if these factors had any influence on the measurements: aortic diameter ( $\leq$ vs $>38 \mathrm{~mm}$ ), valve anatomy (tricuspid vs bicuspid/quadricuspid), stenosis (gradient $\geq 15 \mathrm{~mm} \mathrm{Hg}$ vs $<15 \mathrm{~mm} \mathrm{Hg}$ ) and ROI location (aortic valve vs sinotubular junction).

\section{Statistical analysis}

The qualitative variables were expressed as absolute (n) and relative (\%) frequencies. The Shapiro-Wilk test was applied to evaluate the parametric behaviour of the quantitative variables. Mean values \pm SD are given for normal distributions; for non-normal distributions, the data are reported as medians with IQR.

Pearson's (r) and Spearman's (rho) correlation coefficients were calculated for parametric and non-parametric data, respectively, to determine correlations between the two techniques. For qualitative variables, the Student's t-test or Mann-Whitney U-test (depending on the normality distribution) was applied to analyse differences 


\begin{tabular}{|c|c|}
\hline & $\begin{array}{l}\text { Total } \\
(n=34)\end{array}$ \\
\hline \multicolumn{2}{|l|}{ Gender } \\
\hline Male, n (\%) & $27(79.4)$ \\
\hline Female, n (\%) & $7(20.6)$ \\
\hline Age, median (IQR) & $58.0(17.8)$ \\
\hline \multicolumn{2}{|l|}{ Anatomy } \\
\hline Bicuspid, n (\%) & $12(35.3)$ \\
\hline Tricuspid, n (\%) & $21(61.8)$ \\
\hline Quadricuspid, n (\%) & $1(2.9)$ \\
\hline Diameter $(\mathrm{mm})$, mean $\pm \mathrm{SD}$ & $40.8 \pm 7.4$ \\
\hline \multicolumn{2}{|l|}{ Diameter grouped } \\
\hline$<38 \mathrm{~mm}, \mathrm{n}(\%)$ & $13(38.2)$ \\
\hline$\geq 38 \mathrm{~mm}, \mathrm{n}(\%)$ & $21(61.8)$ \\
\hline \multicolumn{2}{|l|}{ Other pathologies } \\
\hline No pathology, n (\%) & $29(85.4)$ \\
\hline Ascending aortic dissection, $\mathrm{n}(\%)$ & $1(2.9)$ \\
\hline Aortic coarctation (treated), n (\%) & $1(2.9)$ \\
\hline Hypertrophic cardiomyopathy, n (\%) & $2(5.9)$ \\
\hline LV apical infarction with thrombus attached, $\mathrm{n}(\%)$ & $1(2.9)$ \\
\hline LVEF (\%), median (IQR) & $58.0(12.0)$ \\
\hline \multicolumn{2}{|l|}{ LVEF grouped } \\
\hline Decreased, n (\%) & $5(15.2)$ \\
\hline Normal, n (\%) & $28(84.8)$ \\
\hline $\mathrm{EDD}(\mathrm{cm})$, mean $\pm \mathrm{SD}$ & $5.4 \pm 0.9$ \\
\hline \multicolumn{2}{|l|}{ EDD grouped } \\
\hline Dilated, $n(\%)$ & $14(42.4)$ \\
\hline Pathological, n (\%) & $19(57.6)$ \\
\hline
\end{tabular}

EDD, End diastolic diameter; LV, left ventricle; LVEF, Left ventricle ejection fraction.

between the values obtained in the two techniques. The $\chi^{2}$ test or Fisher's exact test was used for qualitative variables. The differences were considered statistically significant for $\mathrm{p}<0.05$. Data analysis was performed with the IBM-SPSS statistical software program, V.21.0.

\section{RESULTS \\ Baseline characteristics}

Thirty-four patients (27 male; 79.4\%) were included in the study. The median age was 58 (17.8) years. The descriptive parameters are shown in table 2 . The mean (SD) maximum diameter of the ascending aorta was 40.8 (7.4) $\mathrm{mm}$. In most cases, the aortic valve anatomy was tricuspid (21 cases; $61.8 \%$ ) or bicuspid $\left({ }^{12} ; 35.3 \%\right)$; one patient $(2.9 \%)$ had a quadricuspid valve. The median LV ejection fraction was 58\% $( \pm 12)$ and the mean enddiastolic diameter of the $\mathrm{LV}$ was $54 \mathrm{~mm}( \pm 9)$.

\section{Comparison between 2DPC MRI and 4D flow MRI}

No statistically significant differences were observed between the two techniques with regard to quantification of forward flow, regurgitant flow or RF (figures 1 and 2).

The severity grading obtained by 2DPC MRI and 4D flow MRI differ in 11 of the 34 patients in our cohort (table 3), with 2DPC MRI overestimating severity compared with 4D flow MRI in 10 of those 11 cases, probably because regurgitant flow and RF values were higher when measured by 2DPC MRI.

There were no significant between-group differences in the quantitative values obtained by the two techniques (figure 3) in any of the subgroup comparisons. Similarly, we found no significant differences between the two subgroups defined according to ROI location (aortic valve vs sinotubular junction) in any of the variables (table 4).

\section{DISCUSSION}

The main aim of this study was to compare 4D flow MRI with 2DPC MRI for the clinical assessment of AR.

For years, 4D flow MRI good internal consistency and good interindividual reproducibility have been well known. ${ }^{22}$ Also, 4D flow MRI has been demonstrated to improve the right ventricular valve flow and diastolic function quantification, ${ }^{23}$ and it has even been used as a reference tool to compare the accuracy of $3 \mathrm{D}$ versus $2 \mathrm{D}$ echography for quantification of AR. ${ }^{24}$

Several authors have validated the use of 4D flow MRI in different clinical situations: to determine morphological and haemodynamic values such as velocity peak and flow displacement, ${ }^{2526}$ to asses severity in hypertrophic myocardiopathy (measuring peak velocity), ${ }^{27}$ to asses dysfunction in bicuspid valves ${ }^{28}$ or to detect incipient haemodynamic changes in Marfan patients. ${ }^{29}$

Even, new automatic techniques for valve tracking have been validated, although not in AR. ${ }^{30}$

To our knowledge, despite the prevalence of AR, there is not a study comparing $4 \mathrm{D}$ flow MRI with 2DPC MRI for grading AR in routine clinical practice. Our aim was to determine if these two imaging modalities are equivalent with regard to AR quantification and if we can confidently change the habitual MRI protocol in this common clinical context.

Importantly, we found no statistically significant differences between the two techniques in the quantitative assessments (figures 1 and 2). For the comparison, 2DPC MRI was used as the reference technique because this sequence has been previously validated by comparison with echocardiography for AR quantification. ${ }^{7-11}$

\section{Flow volume}

Bollache et al found that net volumes measured with the patient in sustained breath-holding are lower than those acquired in, even when the same sequence is used. ${ }^{13}$ Another study showed that 4D flow MRI underestimates individual flow volume quantification compared with 
Positive flow

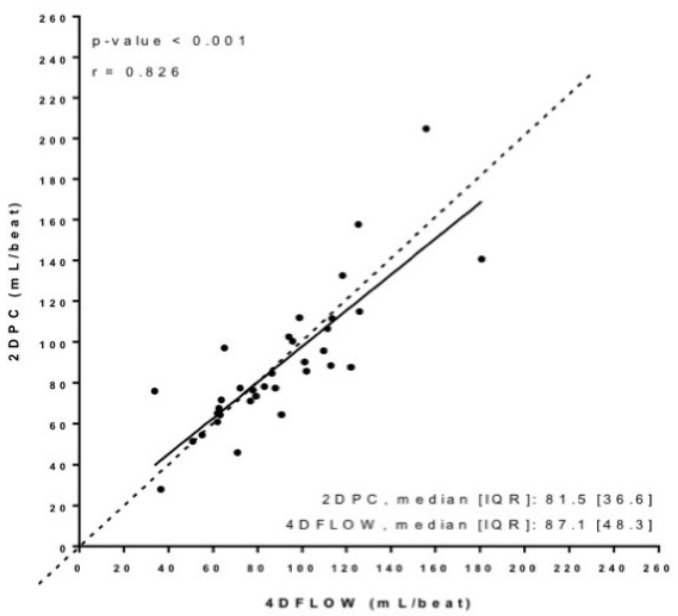

Regurgitant fraction

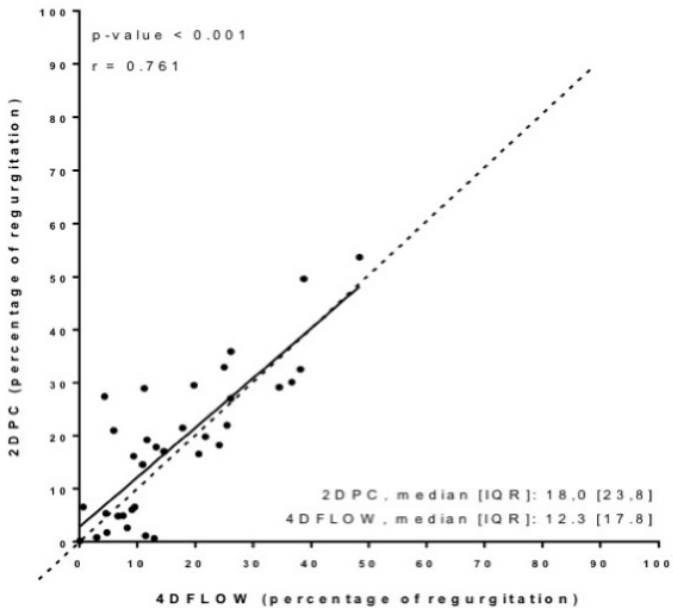

Negative flow
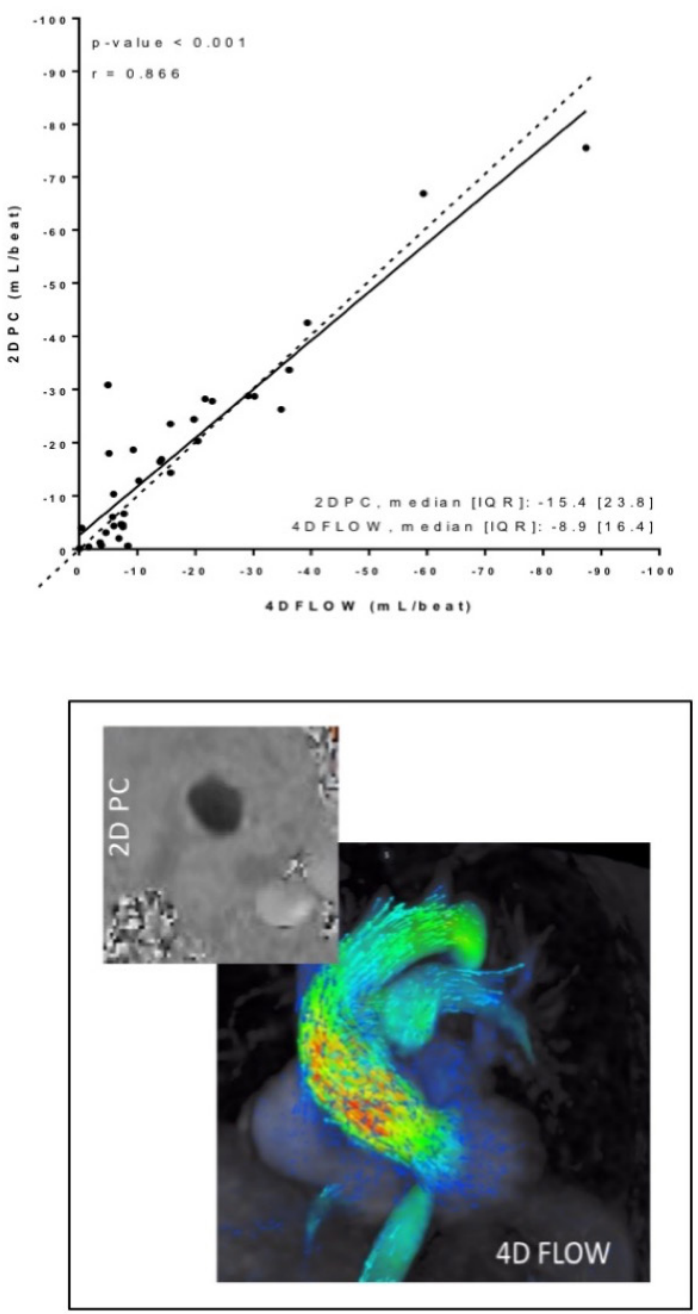

Figure $12 \mathrm{DPC}$ vs 4D flow. Lineal correlation between positive flow, negative flow and RF. Depicts the good correlation between the measurements without statistical differences (2D flow on y-axis) versus 4D flow (x-axis).

2DPC MRI. ${ }^{31}$ In our study, we found no significant differences between 4D flow MRI and 2DPC MRI with regard to forward flow values.

\section{RF and severity grading}

Because RF depends on forward and regurgitant flow, small differences between 2DPC MRI and 4D flow MRI in these volume measurements explains why $\mathrm{RF}$ values measured by 2DPC MRI tend to be higher (figure 2).

Severity criteria for AR assessed by echocardiography, which include qualitative, semiquantitative and quantitative criteria, are well-established in clinical guidelines. ${ }^{1}$ By contrast, the reference values for MRI are less wellestablished and different authors have described different values. For example, some authors have used the same threshold values for MRI as for echocardiography, ${ }^{32}$ while other authors have established different cut-off points. For example, Myerson et $a l^{10}$ concluded that an RF $>33 \%$ predicted the need for valve replacement.

Gelfand $e t a b^{33}$ concluded that the cut-off points for RF that best correlate with echocardiography are as follows: mild AR $(\leq 15 \%)$, moderate $(16 \%-25 \%)$, moderatesevere $(26 \%-48 \%)$ and severe $(>48 \%)$.

If we apply these criteria-which provide narrower ranges that are useful in clinical practice-to our patient data, the severity grading obtained by 2DPC MRI and 4D flow MRI differ in 11 of the 34 patients in our cohort (table 3), with 4D flow MRI underestimating severity compared with 2DPC MRI in 10 of those 11 cases, probably because, as mentioned above, forward flow values tend to be lower when measured by 2DPC MRI (maybe because of the presence of non-laminar flow), whereas the 2DPC MRI regurgitant flow tend to be slightly higher.

Kappa agreement for classifying regurgitation can be calculated between both methods (kappa of 0.72), which means moderate agreement. Also, most of the cases with disagreement would be clinically classified as mildmoderate regurgitation with treatment depending on clinical predictors. Our hypothesis is that with 4D flow MRI, we would use other flow variables (turbulence, jet 
Positive flow

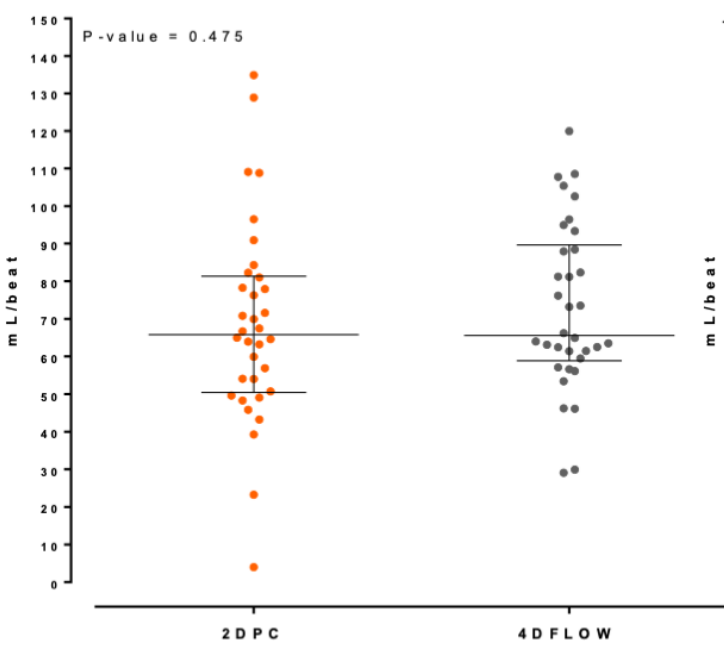

Negative flow

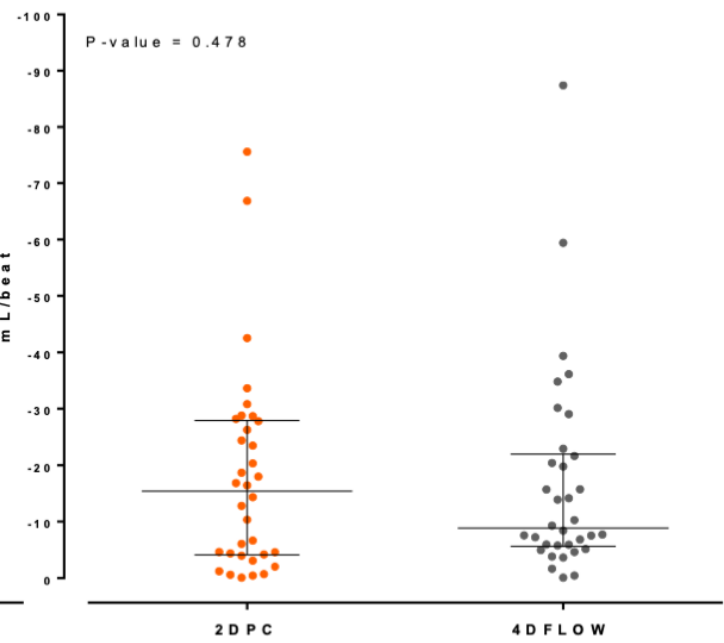

Regurgitan fraction

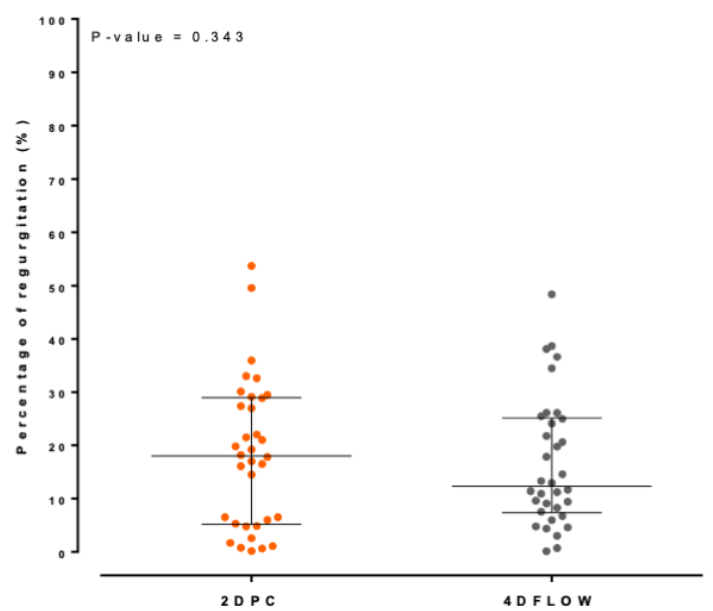

Figure 2 2DPC vs 4Dflow: Comparison of quantitative parameters.

diameter and so on) that improve the AR grading. For this purpose, posterior studies are necessary.

\section{Location of the ROI}

In our study, we used a single double oblique planned acquisition plane orthogonal to the wall of the aorta for the 2DPC MRI acquisition.

However, the optimal location to most accurately measure $\mathrm{AR}$ is controversial ${ }^{19}{ }^{20}$ because $\mathrm{RF}$ values vary depending on the plane that is used. Likewise, the stroke volume is also controversial. Chaturvedi $e t a l^{44}$ found that the most accurate measures of cardiac output are obtained at the proximal ascending aorta; however, Bertelsen et a ${ }^{\beta^{5}}$ found a better correlation for stroke volume at the valve level. For this reason, we compared flow values obtained by placing an ROI in the same location for both MRI sequences (at the valve level or at the sinotubular junction), thus eliminating any possible influence of this variable on the comparative results. Although we found no statistically significant differences in any of the subgroups, the RF values obtained with the two techniques were more closely correlated when the

Table 3 Comparison of AR grading (based on FR) between 2DPC MRI and 4D flow MRI

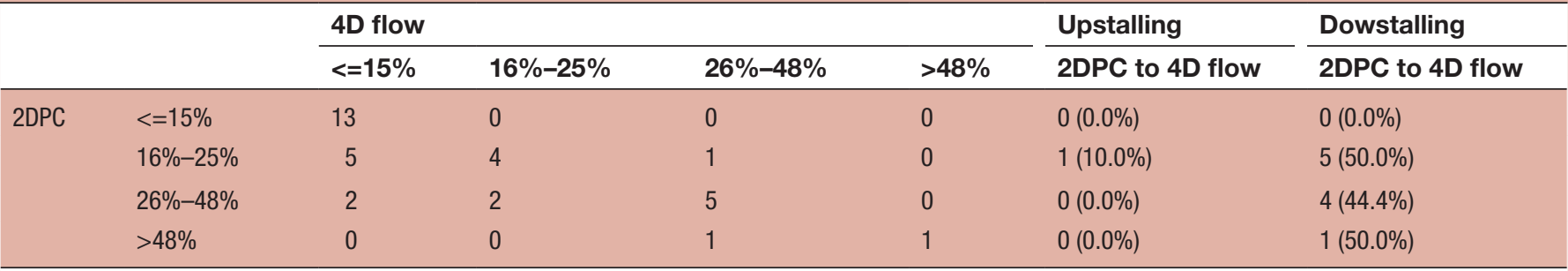

$\mathrm{AR}$, aortic regurgitation; 4D, four dimensional; 2DPC, two-dimensional, phase-contrast . 

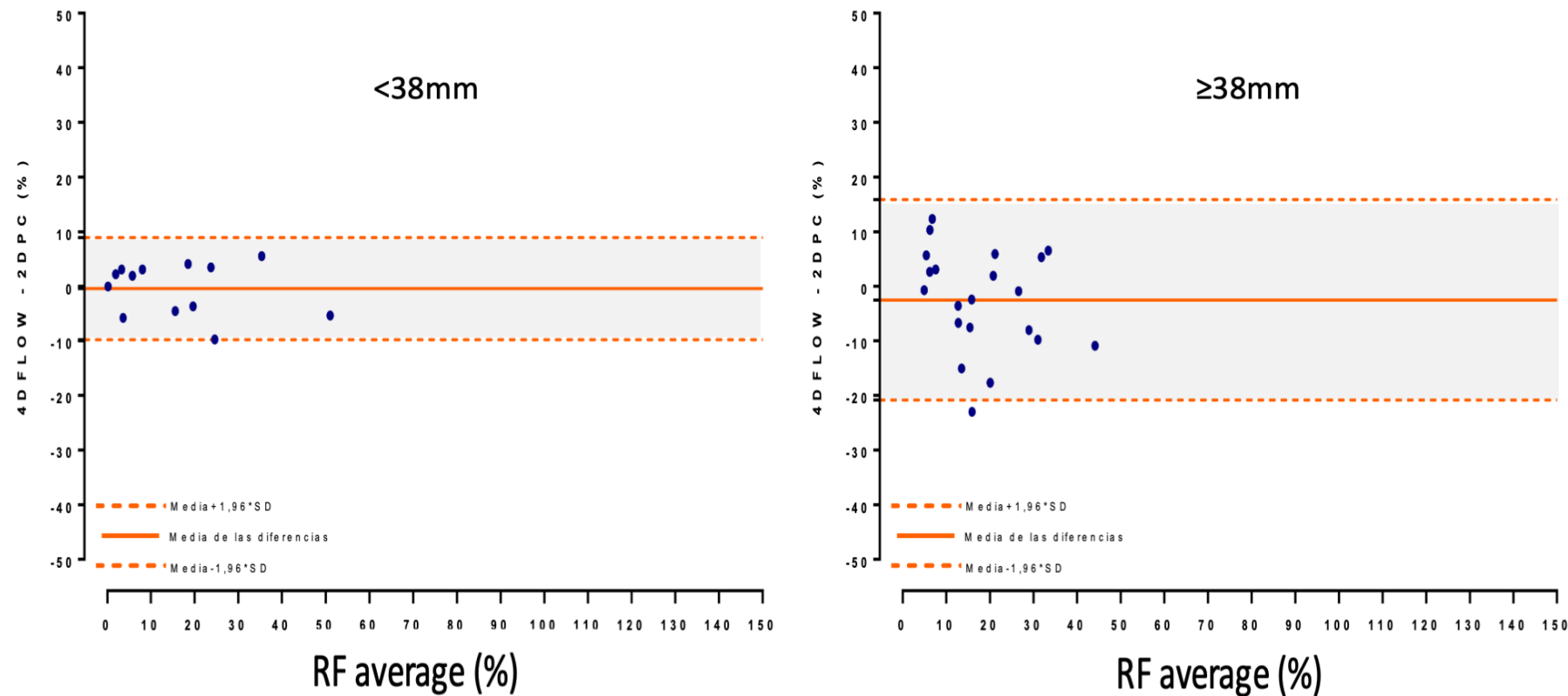

Figure 3 Aortic dilatation. Depicts a Bland-Altman analysis with the difference between both RF measurements (2DPC and 4 Dflow) on the $y$-axis and the average of both measurements on the $x$-axis in each subgroup.

ROI was located at the sinotubular junction (table 4), likely due to the decreased non-laminar flow in this area. This approach does not make use of the possible advantages of 4D flow MRI. We are aware that posterior studies are necessary to determine the best aortic plane for regurgitation quantification with 4D flow MRI for prognostic purposes.

\section{Dilatation}

In patients with dilated aortas and aortas with bicuspid valves, helical flow patterns differ from those observed in healthy patients, resulting in differences in flow measurements. ${ }^{36}$ In this regard, 4D flow MRI is believed to be more accurate than 2DPC MRI in measuring helical flow. ${ }^{38}$ Studies have shown that measurements of cardiac output present substantially more variability in the presence of complex flow patterns secondary to aortic stenosis. ${ }^{39}$ However, we found no significant differences in the values obtained by the two techniques in any of the subgroups analysed (dilated vs non-dilated aortas, tricuspid vs bicuspid/quadricuspid and stenotic vs nonstenotic). RF values were more closely correlated in smaller aortas (diameter $38 \mathrm{~mm}$ ) (figure 3), probably due to non-laminar flow, but we were unable to assess correlations in the other subgroups due to the small number of patients in those groups.

The favourable findings of the present study suggest that the cardiac output values measured by $4 \mathrm{D}$ flow MRI and 2DPC MRI in routine clinical practice do not show statistically significant differences, and in the majority of the patients, the AR grading was concordant.

Also, we suggest that 4D flow MRI has several advantages over 2DPC MRI, particularly with regard to the assessment of complex flows in dilated aortas.

Table 4 ROI location. Correlation coefficients between positive flow, negative flow and RF using 2DPC MRI and 4D flow MRI, depending on the ROI location

\begin{tabular}{|c|c|c|c|c|}
\hline & Valve & Correlation (r) & ST & Correlation (r) \\
\hline & $(n=19)$ & (95\% Cl) & $(n=15)$ & (95\% Cl) \\
\hline \multicolumn{5}{|l|}{ Positive flow (mL/lat) } \\
\hline $2 \mathrm{DPC}$, mean $\pm \mathrm{SD}$ & $96.1 \pm 41.5$ & & $79.4 \pm 18.1$ & \\
\hline \multicolumn{5}{|l|}{ Negative flow (mL/lat) } \\
\hline 2DPC, median (IQR) & $-16.9(24.5)$ & & $-12.8(24.7)$ & \\
\hline \multicolumn{5}{|l|}{ Percentage of regurgitation } \\
\hline 4D flow, median (IQR) & $11.6(20.4)$ & 0.726 (0.394 to 0.891$)$ & $12.9(17.0)$ & 0.861 (0.613 to 0.954$)$ \\
\hline 2DPC, median (IQR) & $18.2(21.7)$ & & $17.0(24.6)$ & \\
\hline
\end{tabular}

4D, four-dimensional; 2DPC, two-dimensional, phase-contrast; RF, regurgitant fraction; ROI, region of interest. 
We believe that 4D flow MRI will, in the future, form part of standard MRI protocols replacing 2DPC MRI. However, additional clinical utility studies are needed to confirm these findings and to confirm the advantages of 4D flow MRI.

The advantages of 4D flow MRI we noticed in our initial practice can be summarised as follows: (1) 3D anatomical, functional and flow data; (2) acquisition during free breathing (2DPC MRI could be acquired with free breathing with navigator-based motion suppression); (3) capacity to retrospectively analyse any flow type (ie, laminar or non-laminar) included in the study, in any direction, compensating the longer duration of the sequence; (4) visualisation of complex or eccentric flows; (5) retrospective tracking of one or more jets to avoid underestimating the $\mathrm{RF}^{40}$; (6) allows assessment of internal validity (eg, by comparing values in the pulmonary artery with those obtained in the aorta); and (7) it can also be used to identify holodiastolic flow reversal in the descending aorta, an independent predictor of severe $\mathrm{AR}^{41}$

\section{Study limitations and strengths}

One of the limitations of this study is the relatively small number of patients. For this reason, larger studies with longer follow-up will be necessary to strengthen the clinical evidence for 4D flow MRI. Another limitation inherent to the two sequences is that 2DPC MRI must be acquired with the patient in breath-hold, whereas $4 \mathrm{D}$ flow acquisition is made in free breathing. An important strength is that this study is the first study to compare $4 \mathrm{D}$ flow MRI for $\mathrm{AR}$ in routine clinical practice with the standard sequence (2DPC MRI).

Furthermore, we expected that 4D flow MRI would permit an accurate diagnosis and provide additional quantitative and qualitative data not obtainable with 2DPC MRI, thus providing a more precise analysis of blood flow, especially in cases with non-laminar or eccentric flow.

\section{CONCLUSIONS}

The findings of this study confirm the value of 4D flow MRI for grading AR in clinical practice. Measures of blood flow and RF obtained with this technique do not differ from those obtained with 2DPC MRI. The present study adds to the growing body of evidence to support the clinical use of 4D flow MRI, which is likely to be included in routine clinical practice in the near future. Indeed, given the numerous advantages of this technique, it may even eventually replace 2DPC MRI in clinical practice. However, further studies are needed to optimise the clinical use of 4D flow MRI.

\section{Twitter Gonzalo Pizarro @GonzaloPiz_Card}

Contributors All authors have made substantial contributions to at least one of the following: the conception and design of the study, or acquisition of data, or analysis and interpretation of data, drafting the article or revising it critically for important intellectual content, final approval of the version to be submitted.
Funding The authors have not declared a specific grant for this research from any funding agency in the public, commercial or not-for-profit sectors.

Competing interests None declared.

Patient consent for publication Not required.

Ethics approval This study was approved by the ethics research committee of the Fundación Jiménez Díaz University Hospital Health Research Institute; CElm-FJD 24 de abril de 2018 (acta $n^{0} 08 / 18$ ).

Provenance and peer review Not commissioned; externally peer reviewed.

Data availability statement Data are available on reasonable request. All of the individual participant data collected will be indefinitely on reasonable request to anyone who wishes access.

Open access This is an open access article distributed in accordance with the Creative Commons Attribution Non Commercial (CC BY-NC 4.0) license, which permits others to distribute, remix, adapt, build upon this work non-commercially, and license their derivative works on different terms, provided the original work is properly cited, appropriate credit is given, any changes made indicated, and the use is non-commercial. See: http://creativecommons.org/licenses/by-nc/4.0/.

ORCID iD

Ana Alvarez http://orcid.org/0000-0002-9554-1541

\section{REFERENCES}

1 Baumgartner H, Falk V, Bax JJ, et al. 2017 ESC/EACTS guidelines for the management of valvular heart disease. Eur Heart $J$ 2017;38:2739-91.

2 Bonow RO, Carabello BA, Kanu C, et al. ACC/AHA 2006 guidelines for the management of patients with valvular heart disease: a report of the American College of Cardiology/American heart association Task force on practice guidelines (writing Committee to revise the 1998 guidelines for the management of patients with valvular heart disease): developed in collaboration with the Society of cardiovascular Anesthesiologists: endorsed by the Society for cardiovascular angiography and interventions and the Society of thoracic surgeons. Circulation 2006;114:e84-231.

3 Detaint D, Messika-Zeitoun D, Maalouf J, et al. Quantitative echocardiographic determinants of clinical outcome in asymptomatic patients with aortic regurgitation: a prospective study. JACC Cardiovasc Imaging 2008;1:1-11.

4 Bellenger Net al. Comparison of left ventricular ejection fraction and volumes in heart failure by echocardiography, radionuclide ventriculography and cardiovascular magnetic resonance. are they interchangeable? Eur Heart J 2000;21:1387-96.

5 Armstrong AC, Gidding S, Gjesdal O, et al. Lv mass assessed by echocardiography and CMR, cardiovascular outcomes, and medical practice. JACC Cardiovasc Imaging 2012;5:837-48.

6 Singer JR. Blood flow rates by nuclear magnetic resonance measurements. Science 1959;130:1652-3.

7 Dulce MC, Mostbeck GH, O'Sullivan M, et al. Severity of aortic regurgitation: interstudy reproducibility of measurements with velocity-encoded cine MR imaging. Radiology 1992;185:235-40.

8 Bakker CJ, Hoogeveen RM, Viergever MA. Construction of a protocol for measuring blood flow by two-dimensional phasecontrast MRA. J Magn Reson Imaging 1999;9:119-27.

9 Cawley PJ, Hamilton-Craig C, Owens DS, et al. Prospective comparison of valve regurgitation quantitation by cardiac magnetic resonance imaging and transthoracic echocardiography. Circ Cardiovasc Imaging 2013;6:48-57.

10 Myerson SG, d'Arcy J, Mohiaddin R, et al. Aortic regurgitation quantification using cardiovascular magnetic resonance: association with clinical outcome. Circulation 2012;126:1452-60.

11 Harris AW, Krieger EV, Kim M, et al. Cardiac magnetic resonance imaging versus transthoracic echocardiography for prediction of outcomes in chronic aortic or mitral regurgitation. Am J Cardiol 2017;119:1074-81.

12 Dyverfeldt P, Bissell M, Barker AJ, et al. 4D flow cardiovascular magnetic resonance consensus statement. J Cardiovasc Magn Reson 2015;17:1-19.

13 Bollache E, van Ooij P, Powell A, et al. Comparison of 4D flow and $2 \mathrm{D}$ velocity-encoded phase contrast MRI sequences for the evaluation of aortic hemodynamics. Int J Cardiovasc Imaging 2016;32:1529-41.

14 Chelu RG, van den Bosch AE, van Kranenburg M, et al. Qualitative grading of aortic regurgitation: a pilot study comparing CMR 4D flow and echocardiography. Int J Cardiovasc Imaging 2016;32:301-7. 
15 Chelu RG, Wanambiro KW, Hsiao A, et al. Cloud-processed 4D CMR flow imaging for pulmonary flow quantification. Eur $J$ Radiol 2016;85:1849-56.

16 Hsiao A, Lustig M, Alley MT, et al. Evaluation of valvular insufficiency and shunts with parallel-imaging compressed-sensing 4D phasecontrast MR imaging with stereoscopic 3D velocity-fusion volumerendered visualization. Radiology 2012;265:87-95.

17 Gabbour M, Rigsby C, Markl M, et al. Comparison of 4D flow and 2D $\mathrm{PC} \mathrm{MRI}$ blood flow quantification in children and young adults with congenital heart disease. J Cardiovasc Magn Reson 2013;15:E90.

18 Podlesnikar T, Delgado V, Bax JJ. Cardiovascular magnetic resonance imaging to assess myocardial fibrosis in valvular heart disease. Int J Cardiovasc Imaging 2018;34:97-112.

19 Gabriel RS, Renapurkar R, Bolen MA, et al. Comparison of severity of aortic regurgitation by cardiovascular magnetic resonance versus transthoracic echocardiography. Am J Cardiol 2011;108:1014-20.

$20 \mathrm{Kim}$ B-G, Kim K-S, Kim S-B, et al. Evaluation of aortic regurgitation by using PC MRI: a comparison of the accuracies at different image plane locations. J Korean Phys Soc 2012;61:1884-8.

21 Rudolph A, Messroghli D, von Knobelsdorff-Brenkenhoff F, et al. Prospective, randomized comparison of gadopentetate and gadobutrol to assess chronic myocardial infarction applying cardiovascular magnetic resonance. BMC Med Imaging 2015;15:1-10.

22 Roes SD, Hammer S, van der Geest RJ, et al. Flow assessment through four heart valves simultaneously using 3-dimensional 3-directional velocity-encoded magnetic resonance imaging with retrospective valve tracking in healthy volunteers and patients with valvular regurgitation. Invest Radiol 2009;44:669-75

23 van der Hulst AE, Westenberg JJM, Kroft LJM, et al. Tetralogy of Fallot: 3D velocity-encoded MR imaging for evaluation of right ventricular valve flow and diastolic function in patients after correction. Radiology 2010;256:724-34.

24 Ewe SH, Delgado V, van der Geest R, et al. Accuracy of threedimensional versus two-dimensional echocardiography for quantification of aortic regurgitation and validation by threedimensional three-directional velocity-encoded magnetic resonance imaging. Am J Cardiol 2013;112:560-6.

25 Garcia J, Barker AJ, van Ooij P, et al. Assessment of altered threedimensional blood characteristics in aortic disease by velocity distribution analysis. Magn Reson Med 2015;74:817-25.

26 Garcia J, Barker AJ, Murphy I, et al. Four-Dimensional flow magnetic resonance imaging-based characterization of aortic morphometry and haemodynamics: impact of age, aortic diameter, and valve morphology. Eur Heart J Cardiovasc Imaging 2016;17:877-84.

27 van Ooij P, Allen BD, Contaldi C, et al. 4D flow MRI and T1 -Mapping: Assessment of altered cardiac hemodynamics and extracellular volume fraction in hypertrophic cardiomyopathy. J Magn Reson Imaging 2016;43:107-14.

28 Geiger J, Rahsepar AA, Suwa K, et al. 4D flow MRI, cardiac function, and $T_{1}$-mapping: Association of valve-mediated changes in aortic hemodynamics with left ventricular remodeling. J Magn Reson Imaging 2018;48:121-31.

29 Geiger J, Hirtler D, Gottfried K, et al. Longitudinal evaluation of aortic hemodynamics in Marfan syndrome: new insights from a 4D flow cardiovascular magnetic resonance Multi-Year follow-up study. $J$ Cardiovasc Magn Reson 2017;19:33.

30 Kamphuis VP, Roest AAW, Ajmone Marsan N, et al. Automated cardiac valve tracking for flow quantification with four-dimensional flow MRI. Radiology 2019;290:70-8.

31 Hanneman K, Sivagnanam M, Nguyen ET, et al. Magnetic resonance assessment of pulmonary (QP) to systemic (QS) flows using 4D phase-contrast imaging: pilot study comparison with standard through-plane 2D phase-contrast imaging. Acad Radiol 2014;21:1002-8.

32 Kawel-Boehm N, Maceira A, Valsangiacomo-Buechel ER, et al. Normal values for cardiovascular magnetic resonance in adults and children. J Cardiovasc Magn Reson 2015;17:1-33.

33 Gelfand EV, Hughes S, Hauser TH, et al. Severity of mitral and aortic regurgitation as assessed by cardiovascular magnetic resonance: optimizing correlation with Doppler echocardiography. J Cardiovasc Magn Reson 2006;8:503-7.

34 Chaturvedi A, Hamilton-Craig C, Cawley PJ, et al. Quantitating aortic regurgitation by cardiovascular magnetic resonance: significant variations due to slice location and breath holding. Eur Radiol 2016;26:3180-9.

35 Bertelsen L, Svendsen JH, Køber L, et al. Flow measurement at the aortic root - impact of location of through-plane phase contrast velocity mapping. J Cardiovasc Magn Reson 2016;18:1-8.

36 Weigang E, Kari FA, Beyersdorf F, et al. Flow-Sensitive fourdimensional magnetic resonance imaging: flow patterns in ascending aortic aneurysms. Eur J Cardiothorac Surg 2008;34:11-16.

37 Bissell MM, Hess AT, Biasiolli L, et al. Aortic dilation in bicuspid aortic valve disease: flow pattern is a major contributor and differs with valve fusion type. Circ Cardiovasc Imaging 2013;6:499-507.

38 Kharabish AE, Belker K, Meierhofer C, et al. Intraindividual validation of $4 \mathrm{D}$ flow measurement against 2D flow measurements in aortas with bicuspid or tricuspid valves by cardiovascular magnetic resonance (CMR). J Cardiovasc Magn Reson 2015;17:P216.

39 Nordmeyer S, Riesenkampff E, Messroghli D, et al. Four-Dimensiona velocity-encoded magnetic resonance imaging improves blood flow quantification in patients with complex accelerated flow. J Magn Reson Imaging 2013;37:208-16.

40 Calkoen EE, Westenberg JJM, Kroft LJM, et al. Characterization and quantification of dynamic eccentric regurgitation of the left atrioventricular valve after atrioventricular septal defect correction with 4D flow cardiovascular magnetic resonance and retrospective valve tracking. J Cardiovasc Magn Reson 2015;17:1-9.

41 Bolen MA, Popovic ZB, Rajiah P, et al. Cardiac Mr assessment of aortic regurgitation: Holodiastolic flow reversal in the descending aorta helps stratify severity. Radiology 2011;260:98-104. 